\title{
SZEGÖ THEOREMS FOR ZOLL OPERATORS
}

\section{Guillemin And K. OKIKIOLU}

The purpose of this note is to announce some new results about multidimensional Szegö estimates in the spirit of $[\mathrm{O}]$. The setting for these results is a compact $d$-dimensional manifold, $X$, and a self-adjoint first order elliptic pseudodifferential operator, $Q: L^{2}(X) \longrightarrow L^{1}(X)$. This operator is called a Zoll operator if its spectrum consists of the integers, $1,2, \ldots$ For such an operator the bicharacteristic flow on the cotangent bundle of $X$ associated with the leading symbol of $Q$ has to be periodic of period $2 \pi$; and to simplify the statements of our results we will assume that this flow is simply periodic of period $2 \pi$, i.e. each bicharacteristic returns for the first time to its initial position at time $t=2 \pi \cdot{ }^{1}$ Let $\pi_{n}$ be projection onto the $n$-th eigenspace of $Q$ and let $P_{n}=\pi_{1}+\cdots+\pi_{n}$. Our main result is the following:

Theorem 1. Let $B$ be a zeroth order pseudodifferential operator for which the symbolic norm of $I-B$ is sufficiently small. Then

$$
\log \operatorname{det}\left(P_{n} B P_{n}\right) \sim b \log n+\sum_{k=d}^{-\infty} b_{k} n^{k}
$$

as $n$ tends to infinity.

The constants, $b$ and $b_{k}, k \neq 0$, are local invariants of $B$, i.e. only depend on the symbol of $B$. In particular the first two are given by the formulas

$$
b_{d}=\frac{1}{d} \operatorname{Res}\left(Q^{-d} \log B\right)
$$

Received June 14, 1996.

The first author is supported by NSF grant DMS-890771.

The second author is supported by NSF grant DMS-9506057.

${ }^{1}$ If we drop this assumption the right hand side of the formula (1) below becomes the sum of a finite number of terms of the form:

$$
\omega_{i}^{n}\left(b_{i} \log n+\sum_{k=d}^{-\infty} b_{i, k} n^{k}\right)
$$

the $\omega_{i}$ 's being roots of unity. 
and

$$
\begin{gathered}
b_{d-1}=\frac{1}{2} \operatorname{Res}\left(Q^{-d} \log B\right)+\frac{1}{d-1} \operatorname{Res}\left(Q^{-(d+1)} \log B\right) \\
+\sum_{j=1}^{\infty} j \operatorname{Res}\left(Q^{-d}(\log B)_{j}(\log B)_{-j}\right)
\end{gathered}
$$

"Res" being the Wodzicki residue. ${ }^{2}$ The most interesting term in this expansion is the non-local term, $b_{0}$, since the exponential of it can be viewed as a regularized determinant of $B$.

We will give a brief sketch of how to prove this result. Expanding $\log P_{n} B P_{n}$ in a Taylor series about the identity it suffices to prove:

Theorem 2. For every zeroth order pseudodifferential operator, A, there exists an asymptotic expansion

$$
\operatorname{trace}\left(P_{n} A P_{n}\right)^{r} \sim a \log n+\sum_{k=d}^{-\infty} a_{k} n^{k}
$$

as $n$ tends to infinity.

Proof. (Sketch) Let $U(t)=\exp i t Q$. The operator $U(-t) A U(t)$ is a periodic function of $t$ of period $2 \pi$; so one can expand it into a Fourier series

$$
\sum A_{k} e^{-i k t}
$$

with

$$
\begin{aligned}
A_{k} & =\frac{1}{2 \pi} \int_{0}^{2 \pi} e^{i k t} U(-t) A U(t) d t \\
& =\sum_{n} \pi_{k+n} A \pi_{n},
\end{aligned}
$$

and Egorov's theorem says that if $A$ is a zeroth order pseudodifferential operator, the $A_{k}$ 's are as well. To prove the theorem, we'll first assume that all but finitely many of them are zero. Letting $\sigma(j)=\max \left(0, j_{1}, \ldots, j_{1}+\right.$ $\left.\cdots+j_{r}\right)$

$$
\operatorname{trace}\left(P_{n} A P_{n}\right)^{r}=\sum_{j_{1}+\cdots+j_{r}=0} \operatorname{trace} \sum_{k+\sigma(j) \leq n} \pi_{k} A_{j_{r}} \ldots A_{j_{1}} \pi_{k},
$$

the number of summands in $j$ being finite. The asymptotic expansion (4) now follows from the following result of Colin de Verdiere:

\footnotetext{
${ }^{2}$ The operators $(\log B)_{j}$ will be defined below.
} 
Lemma 3. For any zeroth order pseudodifferential operator, A,

$$
\operatorname{trace} \pi_{n} A \pi_{n} \sim \sum_{k=d-1}^{-\infty} c_{k}(A) n^{k}
$$

as $n$ tends to infinity.

The $c_{k}(A)$ 's, incidentally, are given by Wodzicki residues

$$
c_{k}(A)=\operatorname{Res}\left(Q^{-(k+1)} A\right)
$$

(c.f. $[\mathrm{GO}]$ ) and hence the terms in the asymptotic expansion (4) are given by Wodzicki residues of products of the Fourier coefficients of $A$.

Finally we prove (4) for arbitrary $A$ by showing that the terms in the Fourier series above are rapidly decreasing as $k$ tends to infinity and hence that $A$ is well-approximated by the finite sum, $\sum_{k=-N}^{N} A_{k}$.

Theorem 4. For every integer, $N$, the operator norm of $A_{k}$ is bounded by $C_{N} k^{-N}, C_{N}$ being a positive constant not depending on $k$.

Proof. Let ad $Q$ be the operation

$$
\text { (ad } Q) A=Q A-A Q .
$$

Then

$$
(\operatorname{ad} Q)^{N} A=\sum k^{N} A_{k}
$$

so the operator norm of $k^{N} A_{k}$ is bounded by the operator norm of $(\operatorname{ad} Q)^{N} A$.

We will conclude by mentioning some other types of multi-dimensional Szegö theorems which can be proved by the methods of this paper:

1. Let $B$ be a zeroth order pseudodifferential operator on $\mathbb{R}^{d}$ whose Weyl symbol is polyhomogeneous with respect to the homotheties, $(x, \xi) \longrightarrow\left(\lambda^{\frac{1}{2}} x, \lambda^{\frac{1}{2}} \xi\right)$ and whose spectrum doesn't contain zero in its convex hull. Letting $P_{N}$ be projection onto the subspace of $L^{2}\left(\mathbb{R}^{d}\right)$ spanned by the Hermite functions of degree $\leq n$, trace $\log \left(P_{n} B P_{n}\right)$ admits a complete asymptotic expansion of the form (1). Moreover, the first two terms in this expansion are given by the formula (2) with $Q$ equal to the harmonic oscillator.

2. Let $B^{d}$ be the closed unit ball in $\mathbb{C}^{d}$ and let $H^{2}$ be the space of $L^{2}$ holomorphic functions on the interior of $B^{d}$. Given $f \in C^{\infty}\left(B^{d}\right)$ let $T_{f}$ be the contraction to $H^{2}$ of the operator, multiplication by $f$, and let $P_{n}$ be the orthogonal projection of $H^{2}$ onto the space spanned by the monomials

$$
z_{1}^{n_{1}} \ldots z_{d}^{n_{d}}, \quad n_{1}+\cdots+n_{d} \leq n .
$$


Then if $f$ has an unambiguously defined logarithm, trace $\log \left(P_{n} T_{f} P_{n}\right)$ admits a complete asymptotic expansion of the form (1).

Some generalizations of this result involving Toeplitz operators on strictly pseudoconvex domains ${ }^{3}$ will be discussed in a future article.

3. The Zoll operator, $Q$, which figures in this paper can be an operator on sections of a vector bundle (providing its leading symbol is of the form, $q I$.

\section{References}

[CV] Y. Colin de Verdiere, Sur le spectre des operateurs elliptiques a bicharacteristiques toutes periodiques, Comm. Math. Helv. 54 (1979), 508-522.

[GO] V. Guillemin and K. Okikiolu, Spectral asymptotics of Toeplitz operators on Zoll manifolds, Preprint.

[O] K. Okikiolu, The analogue of the strong Szegö limit theorem on the 2 and 3 dimensional spheres, J. Amer. Math. Soc., to appear.

[BG] L. Boutet de Monvel and V. Guillemin, The spectral theory of Toeplitz operators, Ann. of Math. Studies, No. 99, Princeton University Press, Princeton, N.J. 1981.

Department of Mathematics, Mit, Cambridge, MA 02139

E-mail address: vwg@math.mit.edu

Department of Mathematics, Mit, Cambridge, MA 02139 and Department of Mathematics-0112, UCSD, LA Jolla, CA 92093-0112

E-mail address: okikiolu@math.mit.edu

\footnotetext{
${ }^{3}$ See $[B G]$. In this reference its shown that the Zoll operators considered here are special examples of such Toeplitz opperators.
} 